TRANSACTIONS OF THE

AMERICAN MATHEMATICAL SOCIETY

Volume 357 , Number 7 , Pages $2557-2569$

S 0002-9947(05)03777-3

Article electronically published on March 1, 2005

\title{
CYCLES ON CURVES OVER GLOBAL FIELDS OF POSITIVE CHARACTERISTIC
}

\author{
REZA AKHTAR
}

\begin{abstract}
Let $k$ be a global field of positive characteristic, and let $\sigma: X \longrightarrow$ Spec $k$ be a smooth projective curve. We study the zero-dimensional cycle group $V(X)=\operatorname{Ker}\left(\sigma_{*}: S K_{1}(X) \rightarrow K_{1}(k)\right)$ and the one-dimensional cycle group $W(X)=\operatorname{coker}\left(\sigma^{*}: K_{2}(k) \rightarrow H_{Z a r}^{0}\left(X, \mathcal{K}_{2}\right)\right)$, addressing the conjecture that $V(X)$ is torsion and $W(X)$ is finitely generated. The main idea is to use Abhyankar's Theorem on resolution of singularities to relate the study of these cycle groups to that of the $K$-groups of a certain smooth projective surface over a finite field.
\end{abstract}

\section{INTRODUCTION}

Let $k$ be a global field of positive characteristic; that is, a field which is finitely generated and of transcendence degree one over a finite field. Let $\sigma: X \longrightarrow$ Spec $k$ be a smooth projective curve over $k$; consider the cycle groups

$$
\begin{gathered}
V(X)=\operatorname{Ker}\left(\sigma_{*}: S K_{1}(X) \rightarrow K_{1}(k)\right), \\
W(X)=\operatorname{Coker}\left(\sigma^{*}: K_{2}(k) \rightarrow H_{Z a r}^{0}\left(X, \mathcal{K}_{2}\right)\right) .
\end{gathered}
$$

In studying $V(X)$ and $W(X)$, it is convenient to use the language of higher Chow groups B13. Using the identifications $C H^{1}(k, 1) \cong K_{1}(k), C H^{2}(k, 2) \cong$ $K_{2}(k), C H^{2}(X, 1) \cong H_{Z a r}^{1}\left(X, \mathcal{K}_{2}\right)=S K_{1}(X)$ and $C H^{2}(X, 2) \cong H_{Z a r}^{0}\left(X, \mathcal{K}_{2}\right)$ and compatibility of the various maps of relevance, one may characterize these groups solely in terms of the higher Chow groups (see [Bl1], [R3], [MSEV]) for details):

$$
\begin{gathered}
V(X)=\operatorname{Ker}\left(\sigma_{*}: C H^{2}(X, 1) \rightarrow C H^{1}(k, 1)\right), \\
W(X)=\operatorname{Coker}\left(\sigma^{*}: C H^{2}(k, 2) \rightarrow C H^{2}(X, 2)\right) .
\end{gathered}
$$

The main conjectures concerning these groups may be enunciated thus:

Conjecture. - (Bloch) $V(X)$ is a torsion group.

- (Raskind) $W(X)$ is a finitely generated group.

In [R3], Raskind has studied $V(X)$ by using Abhyankar's Theorem [Ab] on resolution of singularities for surfaces. Specifically, let $k$ and $X$ be as above, and let $k_{0}$ be the prime field. Choose a smooth projective model $C$ for $k$ over $k_{0}$ and apply Abhyankar's Theorem to deduce the existence of a surface $\mathcal{X}$, smooth and projective over $k_{0}$, together with a proper, flat map $s: \mathcal{X} \rightarrow C$ whose fibers are connected and whose generic fiber is isomorphic to $X$; it is moreover true that for all but finitely many closed points $v \in C$, the special fiber $\mathcal{X}_{v}$ is smooth. To ease notation later in

Received by the editors January 20, 2003.

2000 Mathematics Subject Classification. Primary 14C15, 14C25.

(C)2005 American Mathematical Society 
the paper, set $\mathcal{R}=\left\{v \in C: \mathcal{X}_{v}\right.$ is smooth $\}$ and $\mathcal{S}=\left\{v \in C: \mathcal{X}_{v}\right.$ is not smooth $\}$. In this context, Raskind uses the Sherman exact sequence to prove that $V(X)$ is torsion if $H_{Z a r}^{1}\left(\mathcal{X}, \mathcal{K}_{2}\right)$ is torsion. The two main results of this paper extend Raskind's result and furnish an analogous statement for $W(X)$ :

Theorem. $\bullet V(X)$ is torsion $\Leftrightarrow K_{1}(\mathcal{X})$ is torsion.

- $W(X)$ is finitely generated $\Leftrightarrow K_{2}(\mathcal{X})$ is torsion.

This paper also contains a result giving a rough description of the torsion subgoup of $V(X)$. Moreover, analogous to Raskind's treatment of the number field case [R3], it is proved that $W(X) / n$ is finite for any nonzero integer $n$, giving further evidence towards the above conjecture.

Throughout this paper an algebraic scheme is a scheme of finite type over a field $k$, while a variety is an integral algebraic scheme. An integral algebraic scheme is called a curve if it is one dimensional or a surface if it is two dimensional. If $x \in X$ is any point, the residue field at $x$ is denoted $k(x)$; if $X$ happens to be irreducible, the residue field at the generic point of $X$ (i.e. the function field) is written $k(X)$. If $X \longrightarrow$ Spec $k$ is an algebraic scheme and $L / k$ is a field extension, we write $X_{L}$ for the base extension $X \times{ }_{\text {Spec }}{ }_{k} \operatorname{Spec} L$.

For any scheme $X$, the notation $K_{*}(X)$ refers to the algebraic $K$-theory of $X$. If $X$ is an algebraic scheme, $C H^{*}(X)$ denotes the Chow ring of $X$ and $C H^{*}(X, \cdot)$ the higher Chow groups of $X$. We often write $C H^{*}(k, \cdot)$ instead of $C H^{*}(\operatorname{Spec} k, \cdot)$. If $G$ is an abelian group and $n>0$ an integer, we use the shorthand ${ }_{n} G$ for the $n$-torsion subgroup of $G$ and $G / n$ for the quotient $G / n G$. The group of $n$th roots of unity in (a field) $k$ is denoted $\mu_{n}(k)$.

The author thanks the College of Arts and Sciences at Miami University for funding this research during the summer of 2002, and the referee for several helpful suggestions.

\section{THE $K$-THEORY OF $X$}

To motivate the study of the groups $V(X)$ and $W(X)$, consider the problem of describing $K_{i}(X)$ for $i \geq 0$. It is well known that $K_{0}(X) \otimes \mathbb{Q} \cong \mathbb{Q} \oplus(\operatorname{Pic}(X) \otimes \mathbb{Q})$; however, little is known in general when $i>0$. By using Abhyankar's construction, we may try to reduce the study of cycles on $X$, the generic fiber of the map $s$ : $\mathcal{X} \longrightarrow C$ to the study of cycles on $\mathcal{X}$ itself and cycles on the various special fibers $\mathcal{X}_{v}$. To make any progress, though, we either have to make some fairly restrictive assumptions on $\mathcal{X}$ or assume the following conjecture of Parshin.

Conjecture 2.1. Let $Y$ be a smooth projective variety over a finite field. Then the groups $K_{i}(Y)$ are torsion when $i>0$.

We remark in passing that a conjecture attributed to Bass predicts that all the groups $K_{i}(Y)$ for $i \geq 0$ are finitely generated; together, the two conjectures imply that $K_{i}(Y)$ is actually finite for $i>0$.

Probably the most significant progress towards verification of Parshin's conjecture has been made by Soulé Sou. For any field $F$, he describes a class $A(F)$ of smooth projective varieties, closed under products and containing all curves over $F$ and unirational varieties of degree $\geq 3$. Soulé then proves the following theorem. 
Theorem 2.2 ([Sou], Théorème 6). Suppose $F$ is a finite field. If $Y$ is in the class $A(F)$, then $K_{0}(Y)$ is finitely generated, and if $\operatorname{dim} Y \leq 3$, then $K_{m}(Y)$ has finite exponent for all $m \geq 1$.

Conjecturally, of course, $A(F)$ contains all smooth projective varieties over $F$.

In dimension 1 , the structure of $K_{1}$ is understood completely:

Theorem 2.3 (Nestler, $\mathrm{Ne}], 3.1$ Theorem 3). Let $Y$ be a smooth projective reduced one-dimensional algebraic scheme over a finite field with $r$ irreducible components. Then $K_{1}(Y) \cong k^{*} \oplus\left(k^{*}\right)^{r}$.

Because some of the fibers $\mathcal{X}_{v}$ may not be smooth, we need the following:

Lemma 2.4. Let $F$ be a finite field and $\Gamma$ a (not necessarily smooth) projective curve over $F$. Then $K_{0}(\Gamma)$ and $K_{1}(\Gamma)$ are finitely genereated, and $K_{m}(\Gamma)$ is of finite exponent when $m \geq 2$.

Proof. Each of these statements follows from the localization sequence for algebraic $K$-theory Qu2 by comparing $\Gamma$ to its normalization. Precisely, let $\nu: \tilde{\Gamma} \longrightarrow \Gamma$ be the normalization of $\Gamma$ and let $U \subseteq \Gamma$ be an open subset such that $\left.\nu\right|_{\nu^{-1}(U)}$ : $\nu^{-1}(U) \longrightarrow U$ is an isomorphism. Set $Z=\Gamma-U, \tilde{U}=\nu^{-1}(U)$ and $\tilde{Z}=\nu^{-1}(Z)$. Then we have the following commutative diagram with exact rows, in which the vertical arrows are induced by $\nu$ :

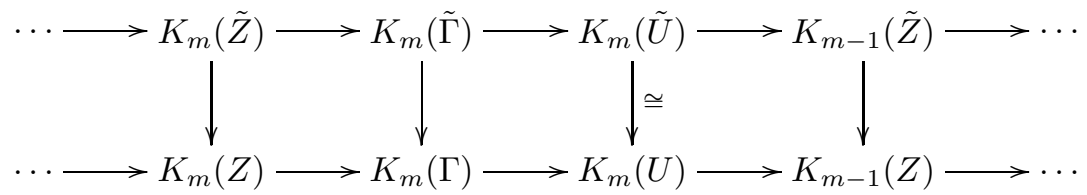

Note that $Z$ (respectively $\tilde{Z}$ ) is a finite set of closed points. Thus, $K_{0}(Z)$ (resp. $\left.K_{0}(\tilde{Z})\right)$ is a finitely generated free abelian group. Using Quillen's calculation of the $K$-theory of finite fields Qu1, we see that $K_{r}(Z)\left(\right.$ resp. $\left.K_{r}(\tilde{Z})\right)$ is a finite group for all $r \geq 1$. The conclusion follows easily from these remarks and the characterization of the $K$-groups of (the smooth projective curve) $\tilde{\Gamma}$ from Theorems 2.2 and 2.3 .

The main result of relevance to us is the following:

Proposition 2.5. Assume Parshin's conjecture. Then $K_{m}(X)$ is a torsion group for $m \geq 3$, and $K_{2}(X)$ has finite rank.

Proof. By Parshin's conjecture, the $K$-groups $K_{m}(\mathcal{X})$ are torsion for $m \geq 1$. Localization gives an exact sequence:

$$
\cdots \longrightarrow K_{m}(\mathcal{X}) \longrightarrow K_{m}(X) \longrightarrow \bigoplus_{v \in C} K_{m-1}\left(\mathcal{X}_{v}\right) \longrightarrow
$$

If $m \geq 3$, then all the groups $K_{m-1}\left(\mathcal{X}_{v}\right)$ are torsion groups by Lemma2.4 therefore $K_{m}(X)$, being sandwiched between two torsion groups in the exact sequence, must itself be torsion. If $m=2$, then the groups $K_{1}\left(\mathcal{X}_{v}\right)$ corresponding to smooth fibers are torsion by Theorem 2.3 , the groups corresponding to singular fibers have finite rank by Lemma 2.4. Since there are only finitely many $v$ of the latter type, we see that $K_{2}(X)$ is sandwiched between the torsion group $K_{2}(\mathcal{X})$ and the group $\bigoplus_{v \in C} K_{1}\left(\mathcal{X}_{v}\right)$ of finite rank; hence it, too, has finite rank. 
The connection between the $K$-theory of $X$ and the groups $V(X)$ and $W(X)$ is apparent from the well-known "Riemann-Roch" theorem of Bloch [Bl3], which will be used in later sections:

Theorem 2.6. Let $Y$ be an algebraic scheme. Then there is an isomorphism

$$
G_{n}(Y) \otimes \mathbb{Q} \cong \bigoplus_{i} C H^{i}(Y, n) \otimes \mathbb{Q},
$$

where $G_{n}(Y)$ are the higher $K$-groups of the category of coherent sheaves on $Y$ as defined by Quillen Qu2].

In particular, if $Y$ is smooth, then

$$
K_{n}(Y) \otimes \mathbb{Q} \cong \bigoplus_{i} C H^{i}(Y, n) \otimes \mathbb{Q} .
$$

As an example of Shapiro ([Sha, Prop. 2) shows, the analogue of this theorem with integral coefficients is not valid; the integral version of this theorem is the so-called Bloch-Lichtenbaum spectral sequence ([BL] and [FS]), the Riemann-Roch theorem being its degenerate form upon tensoring with $\mathbb{Q}$.

Returning to the context of the discussion, Theorem 2.6 explains why one cannot hope for $K_{1}(X)$ to be torsion or even of finite rank:

$$
K_{1}(X) \otimes \mathbb{Q} \cong \bigoplus_{i} C H^{i}(X, 1) \otimes \mathbb{Q} .
$$

When $i>2$ or $i<0, C H^{i}(X, 1)=0$ for reasons of dimension. Furthermore, we have $C H^{0}(X, 1)=0$, and $C H^{1}(X, 1) \cong k^{*}$ by $\mathrm{Bl3}$, Theorem 6.1. Thus $K_{1}(X) \otimes \mathbb{Q}$ already contains a (clearly identified) subgroup of infinite rank. Moreover, the conjecture that $V(X)$ is torsion implies that $C H^{2}(X, 1) \otimes \mathbb{Q} \cong k^{*} \otimes \mathbb{Q}$, and so $K_{1}(X) \otimes \mathbb{Q} \cong\left(k^{*} \oplus k^{*}\right) \otimes \mathbb{Q}$.

\section{The group $V(X)$}

Our first goal is to prove:

Theorem 3.1. $V(X)$ is torsion $\Leftrightarrow K_{1}(\mathcal{X})$ is torsion.

Observe that by the Riemann-Roch Theorem (Theorem 2.6), we have

$$
K_{1}(\mathcal{X}) \otimes \mathbb{Q} \cong \bigoplus_{i} C H^{i}(\mathcal{X}, 1) .
$$

Since $\mathcal{X}$ is a surface, $C H^{i}(\mathcal{X}, 1)=0$ when $i>3$ or $i \leq 0$ for reasons of dimension. Moreover, $C H^{0}(\mathcal{X}, 1)=0$. Also, $C H^{1}(\mathcal{X}, 1)=k_{0}^{*}$, which is a torsion group, and $C H^{3}(\mathcal{X}, 1)$ is a torsion group by [Ak2], Theorem 3.1. Thus, to prove Theorem 3.1 it suffices to show that $V(X)$ is torsion if and only if $C H^{2}(\mathcal{X}, 1)=H_{Z a r}^{1}\left(\mathcal{X}, \mathcal{K}_{2}\right)$ is torsion.

We begin with a result of Raskind whose proof has been simplified somewhat by using the language of higher Chow groups; together with the discussion of the previous paragraph, this takes care of the "if" statement of the theorem.

First, we give a new construction of an (extension of an) exact sequence due to Sherman She]. 
Proposition 3.2. There is an exact sequence

$$
\begin{gathered}
0 \longrightarrow H_{Z a r}^{0}\left(\mathcal{X}, \mathcal{K}_{2}\right) \longrightarrow H_{Z a r}^{0}\left(X, \mathcal{K}_{2}\right) \longrightarrow \bigoplus_{v \in C} C H^{1}\left(\mathcal{X}_{v}, 1\right) \longrightarrow H_{Z a r}^{1}\left(\mathcal{X}, \mathcal{K}_{2}\right) \\
\longrightarrow H_{Z a r}^{1}\left(X, \mathcal{K}_{2}\right) \longrightarrow \bigoplus_{v \in C} \text { Pic } \mathcal{X}_{v} \longrightarrow C H^{2}(\mathcal{X}) \longrightarrow 0
\end{gathered}
$$

Proof. This is simply the localization sequence for higher Chow groups, taking into account the fact that for any quasiprojective algebraic scheme $Y, C H^{1}(Y, 2)=0$ and $C H^{2}(Y, 0)=C H^{2}(Y)$ [Bl3], and for $Y$ smooth, $C H^{1}(Y, 0) \cong C H^{1}(Y) \cong$ $\operatorname{Pic}(Y)(\mathrm{Bl3}]), C H^{2}(Y, 2) \cong H_{Z a r}^{0}\left(Y, \mathcal{K}_{2}\right)$, and $C H^{2}(Y, 1) \cong H_{Z a r}^{1}\left(Y, \mathcal{K}_{2}\right)$, as described in the Introduction.

Proposition 3.3 (Raskind, [R3], Lemma 2.2). If $H_{Z a r}^{1}(\mathcal{X}, 2)$ is a torsion group, then $V(X)$ is a torsion group. In particular, if $X$ is in the class $A\left(k_{0}\right)$, then $V(X)$ is a torsion group.

Remark. Raskind also shows that if $H_{Z a r}^{1}(\mathcal{X}, 2)$ is not a torsion group, then $V(X)$ contains an infinite divisible subgroup, although we will not make use of this result.

Proof. Covariant functoriality of the higher Chow groups with respect to the (proper) map $s: \mathcal{X} \longrightarrow C$ gives the commutative diagram in which the rows are exact, coming from the various localization sequences (to ease notation, the vertical maps induced by $s$ are all labeled $s_{*}$, although they are obviously different maps):

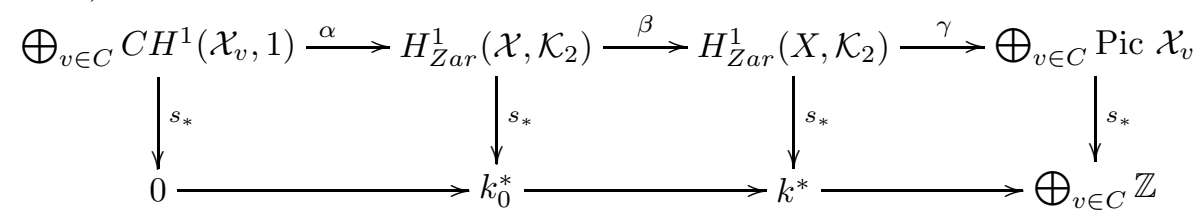

Suppose $c \in V(X)$ is some element. By commutativity of the middle square, it follows that

$$
\gamma(c) \in \operatorname{Ker}\left(s_{*}: \bigoplus_{v \in C} \operatorname{Pic} \mathcal{X}_{v} \longrightarrow \bigoplus_{v \in C} \mathbb{Z}\right) \cong \bigoplus_{v \in C} \operatorname{Ker}\left(s_{*}: \operatorname{Pic} \mathcal{X}_{v} \longrightarrow \mathbb{Z}\right) .
$$

Since all the fibers $\mathcal{X}_{v}$ are connected, the group on the right is a torsion group. Thus, $\gamma(c)$ is torsion and hence some multiple of $c$ is in $\operatorname{Im} \beta$. However, since $H_{Z a r}^{1}\left(\mathcal{X}, \mathcal{K}_{2}\right)$ is torsion, it follows immediately that $\operatorname{Im} \beta$ is torsion. Thus $c$ itself is a torsion element.

Corollary 3.4. Suppose $X=\Gamma \times_{k_{0}} k$, where $\Gamma$ is a smooth projective curve over $k_{0}$. Then $V(X)$ is a torsion group.

Proof. In this case, $\mathcal{X} \cong \Gamma \times_{k_{0}} C$; by Theorem [2.2, $K_{1}(\mathcal{X})$ is a torsion group hence by Riemann-Roch the same is true of $H_{Z a r}^{1}\left(\mathcal{X}, \mathcal{K}_{2}\right)$.

We now turn our attention to the "only if" statement of Theorem 3.1

Recall that $\mathcal{R}$ is the set of $v \in C$ such that the (special) fiber $\mathcal{X}_{v}$ is smooth. For such $v$, we have $C H^{1}\left(\mathcal{X}_{v}, 1\right) \cong k(v)^{*}$ [Bl3]. In general, we have:

Lemma 3.5. Let $v \in C$ be a closed point. Then $C H^{1}\left(\mathcal{X}_{v}, 1\right)$ is a finitely generated group. 
Proof. For convenience of notation, set $V=\mathcal{X}_{v}$ and let $\nu: \tilde{V} \longrightarrow V$ denote the normalization map. Choose an open set $U \subseteq V$ such that $\left.\nu\right|_{\nu^{-1}(U)}: \nu^{-1}(U) \longrightarrow U$ is an isomorphism and set $Z=V-U, \bar{Z}=\tilde{V}-\nu^{-1}(U)$. Then localization gives a commutative diagram with exact rows; we are implicitly using the fact that $C H^{0}(Y, 1)=0$ vanishes for any algebraic scheme $Y$.

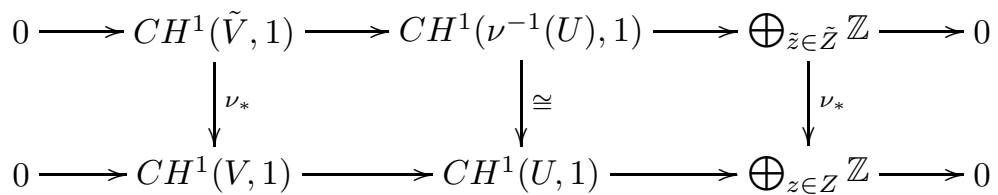

Since $\tilde{V}$ is smooth, we have $C H^{1}(\tilde{V}, 1) \cong k(v)^{*}$ which is finite. Observing that the sets $Z$ and $\tilde{Z}$ are finite allows us to conclude that $C H^{1}(V, 1)$ is finitely generated.

The following result on the structure of $H_{Z a r}^{1}\left(\mathcal{X}, \mathcal{K}_{2}\right)$ will be of crucial importance:

Lemma 3.6 (Gros and Suwa, [GS], Theorem 4.19). Let $F$ be a finite field, $p=$ char $F$, and $Y$ a smooth projective variety over $F$. There is an exact sequence:

$$
0 \longrightarrow T \longrightarrow H_{Z a r}^{1}\left(Y, \mathcal{K}_{2}\right) \longrightarrow \bigoplus_{l \neq p} H_{e t}^{3}\left(Y, \mathbf{Z}_{l}(2)\right) \longrightarrow 0
$$

in which $T$ is a uniquely divisible group and the group on the right is finite.

Proposition 3.7. With notation as in the Sherman exact sequence, Im $\alpha=\operatorname{Ker} \beta$ is a finitely generated group.

Proof. We now use contravariant functoriality of the higher Chow groups with respect to the morphism $s: \mathcal{X} \longrightarrow C$. Localization once again gives a commutative diagram with exact rows (again, the vertical maps are all labelled $s^{*}$ for the sake of notational simplicity):

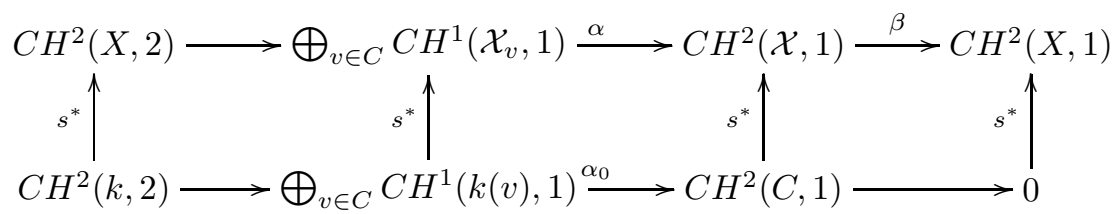

Observe that

$$
\operatorname{Im} \alpha=\sum_{v \in \mathcal{R}} \alpha\left(C H^{1}\left(\mathcal{X}_{v}, 1\right)\right)+\sum_{v \in \mathcal{S}} \alpha\left(C H^{1}\left(\mathcal{X}_{v}, 1\right)\right) .
$$

However, for $v \in \mathcal{R}, s^{*}$ is an isomorphism, so

$$
\alpha\left(C H^{1}\left(\mathcal{X}_{v}, 1\right)\right)=\alpha\left(s^{*}\left(C H^{1}(k(v), 1)\right)\right)=s^{*}\left(\alpha_{0}\left(C H^{1}(k(v), 1)\right)\right) .
$$

This, however, is contained in $s^{*}\left(C H^{2}(C, 1)\right)$, which is finite in view of the isomorphism $C H^{2}(C, 1) \cong k_{0}^{*}$ ( $\mathrm{Ak} 2$, Theorem 3.1).

Now for the (finitely many) $v \in \mathcal{S}$, it only remains to observe that $C H^{1}\left(\mathcal{X}_{v}, 1\right)$ is finitely generated by Lemma 3.5 hence the same must be true of $\alpha\left(C H^{1}\left(\mathcal{X}_{v}, 1\right)\right)$. 
Proof of Theorem 3.1. Assume that $V(X)$ is torsion.

Commutativity of the middle square of the diagram appearing in the proof of Proposition 3.3 shows that $s_{*}(\operatorname{Im} \beta)$ is contained in $k_{0}^{*}$, which is a finite group. Hence, if $m=\left|k_{0}^{*}\right|$, then $m(\operatorname{Im} \beta) \subseteq V(X)$ and so $\operatorname{Im} \beta$ is a torsion group. Because Ker $\beta$ is finitely generated by Proposition 3.7, it follows that $H_{Z a r}^{1}\left(\mathcal{X}, \mathcal{K}_{2}\right)$ has finite rank. However, Lemma 3.6 implies that $H_{Z a r}^{1}\left(\mathcal{X}, \mathcal{K}_{2}\right)$ contains a uniquely divisible subgroup $T$, and in order for this subgroup to be nontrivial, it must have infinite rank. Thus $T=0$ and so $H_{Z a r}^{1}\left(\mathcal{X}, \mathcal{K}_{2}\right)$ is finite, hence torsion.

We conclude this section by giving a partial description of the torsion subgroup of $V(X)$, which will typically turn out to be quite large. The following result of Colliot-Thélène, Sansuc, and Soulé will be helpful:

Theorem 3.8 (CTSS, Theorem 3.7). Let $Y$ be a smooth projective variety over a finite field. Then the torsion subgroup of $\mathrm{CH}^{2}(Y)$ is finite.

It is worth mentioning that the above result may also be deduced from a theorem of Kato and Saito [KS] which states that for any such $Y$ as above, $C H_{0}(Y) \cong$ $\mathbb{Z} \oplus A_{0}(Y)$, where $A_{0}(Y)$ is a finite group.

Proposition 3.9. Let $r=r k(\operatorname{Im} \alpha)$. There exists an integer $N$ such that for all $m$ prime to $N$, there is an exact sequence

$$
0 \longrightarrow(\mathbb{Z} / m \mathbb{Z})^{r} \longrightarrow H_{Z a r}^{1}\left(X, \mathcal{K}_{2}\right) \longrightarrow \bigoplus_{v \in C}{ }_{m} \text { Pic } \mathcal{X}_{v} \longrightarrow 0
$$

Proof. We first examine the end of the Sherman exact sequence (Proposition 3.2):

$$
H_{Z a r}^{1}\left(\mathcal{X}, \mathcal{K}_{2}\right) \stackrel{\beta}{\rightarrow} H_{Z a r}^{1}\left(X, \mathcal{K}_{2}\right) \stackrel{\gamma}{\rightarrow} \bigoplus_{v \in C} \operatorname{Pic} \mathcal{X}_{v} \stackrel{\epsilon}{\rightarrow} C H^{2}(\mathcal{X}) \rightarrow 0 .
$$

Multiplication by $m$ gives a map from the exact sequence

$$
0 \longrightarrow \operatorname{Ker} \epsilon \longrightarrow \bigoplus_{v \in C} \operatorname{Pic} \mathcal{X}_{v} \stackrel{\epsilon}{\longrightarrow} C H^{2}(\mathcal{X}) \longrightarrow 0
$$

to itself; thus the snake lemma yields an exact sequence

$$
\begin{gathered}
0 \rightarrow{ }_{m}(\operatorname{Ker} \epsilon) \rightarrow \bigoplus_{v \in C}{ }_{m}\left(\operatorname{Pic} \mathcal{X}_{v}\right) \rightarrow{ }_{m} C H^{2}(\mathcal{X}) \rightarrow(\text { Ker } \epsilon) / m \\
\rightarrow \bigoplus_{v \in C} \operatorname{Pic} \mathcal{X}_{v} / m \rightarrow C H^{2}(\mathcal{X}) / m \longrightarrow 0
\end{gathered}
$$

By Theorem 3.8 the third term is zero for $m$ prime to the order of the torsion subgroup of $\mathrm{CH}^{2}(\mathcal{X})$. Hence for such $m$ there is an isomorphism

$$
{ }_{m}(\operatorname{Ker} \epsilon) \cong \bigoplus_{v \in C} m\left(\operatorname{Pic} \mathcal{X}_{v}\right)
$$

Furthermore, the exact sequence

$$
0 \longrightarrow \operatorname{Ker} \beta \longrightarrow H_{Z a r}^{1}\left(\mathcal{X}, \mathcal{K}_{2}\right) \longrightarrow \operatorname{Ker} \gamma \longrightarrow 0
$$

gives rise to another exact sequence,

$$
\begin{gathered}
0 \rightarrow m(\operatorname{Ker} \beta) \rightarrow{ }_{m} H_{Z a r}^{1}\left(\mathcal{X}, \mathcal{K}_{2}\right) \rightarrow m(\operatorname{Ker} \gamma) \rightarrow(\operatorname{Ker} \beta) / m \\
\rightarrow H_{Z a r}^{1}\left(\mathcal{X}, \mathcal{K}_{2}\right) / m \rightarrow(\operatorname{Ker} \gamma) / m \longrightarrow 0
\end{gathered}
$$


By Lemma 3.6, it follows that the second and fifth terms are 0 for $m$ prime to some sufficiently large integer. Hence, for such $m$, we have

$$
{ }_{m}(\operatorname{Ker} \gamma) \cong(\operatorname{Ker} \beta) / m \text { and }(\operatorname{Ker} \gamma) / m=0 .
$$

Once again, the short exact sequence

$$
0 \longrightarrow \operatorname{Ker} \gamma \longrightarrow H_{Z a r}^{1}\left(X, \mathcal{K}_{2}\right) \longrightarrow \text { Ker } \epsilon \longrightarrow 0
$$

induces a long exact sequence:

$$
\begin{gathered}
0 \rightarrow{ }_{m}(\operatorname{Ker} \gamma) \rightarrow{ }_{m} H_{Z a r}^{1}\left(X, \mathcal{K}_{2}\right) \rightarrow m(\operatorname{Ker} \epsilon) \rightarrow(\operatorname{Ker} \gamma) / m \\
\rightarrow H_{Z a r}^{1}\left(X, \mathcal{K}_{2}\right) / m \rightarrow(\operatorname{Ker} \epsilon) / m \longrightarrow 0
\end{gathered}
$$

Using the isomorphisms noted, the above condenses to a short exact sequence

$$
0 \longrightarrow(\operatorname{Im} \alpha) / m \longrightarrow{ }_{m} H_{Z a r}^{1}\left(X, \mathcal{K}_{2}\right) \longrightarrow \bigoplus_{v \in C} m\left(\operatorname{Pic} \mathcal{X}_{v}\right) \longrightarrow 0
$$

Finally, taking $m$ to be relatively prime to the order of the torsion subgroup of $\operatorname{Im} \alpha$, we conclude that $(\operatorname{Im} \alpha) / m \cong(\mathbb{Z} / m \mathbb{Z})^{r}$, finishing the proof of Proposition 3.9 .

Remark. Since $V(X)=\operatorname{Ker}\left(\sigma_{*}: H_{Z a r}^{1}\left(X, \mathcal{K}_{2}\right) \rightarrow k^{*}\right)$, there is always an exact sequence

$$
0 \longrightarrow{ }_{m} V(X) \rightarrow{ }_{m} H_{Z a r}^{1}\left(X, \mathcal{K}_{2}\right) \rightarrow{ }_{m} \operatorname{Im} \sigma_{*} .
$$

(If $X$ has a $k$-rational point, then the rightmost map is in fact split surjective.) In any case, since ${ }_{m} \operatorname{Im} \sigma_{*} \subseteq{ }_{m} k^{*}=\mu_{m}(k)$ is finite, it is clear that ${ }_{m} V(X)$ is typically quite large, too.

\section{The group $W(X)$}

Raskind [R1] has studied the group $W(X)=H_{Z a r}^{0}\left(X, \mathcal{K}_{2}\right) / K_{2} k$ when $k$ is a number field. The restriction to characteristic zero is probably due to the fact that the tools he used (explicated in detail in [R2]) were not yet available in positive characteristic at the time of writing. As in the previous section, we work in the setting of positive characteristic, using different techniques to prove an analogue of Raskind's "weak Mordell-Weil theorem for $W(X)$ ", that is, finiteness of $W(X) / n$ for any integer $n$.

We will need another result of Gros and Suwa, the analogue of Lemma 3.6 for $H_{Z a r}^{0}\left(Y, \mathcal{K}_{2}\right)$.

Lemma 4.1 (Gros and Suwa, GS, Theorem 4.11). Let $F$ be a finite field of characteristic $p$ and $Y$ a projective variety, smooth over $F$. Then there is an exact sequence

$$
0 \longrightarrow T \longrightarrow H_{Z a r}^{0}\left(Y, \mathcal{K}_{2}\right) \longrightarrow \bigoplus_{l \neq p} H_{e t}^{2}\left(Y, \mathbb{Z}_{l}(2)\right) \longrightarrow 0
$$

in which the group on the right is finite and $T$ is a uniquely divisible group.

Ultimately, we will need a result of Bass and Tate which appears in a different guise in $[\mathrm{BT}]$ :

Theorem 4.2 (Bass and Tate, [BT], II. Theorem 2.1). Let $Z$ be a curve, smooth and projective over a finite field. Then $C H^{2}(Z, 2)$ is a finite group. 
(Bass and Tate prove that the "tame kernel"

$$
\operatorname{Ker}\left(\partial: K_{2}^{M} k(Z) \longrightarrow \bigoplus_{v \in Z} K_{1}^{M} k(v)\right)
$$

is a finite group; an easy argument using localization sequences identifies this group with $C H^{2}(Z, 2)$.)

Proposition 4.3 ("Weak Mordell-Weil Theorem for $W(X)$ "). For all nonzero integers $n$, the group $W(X) / n$ is finite.

Proof. From the contravariant structure of higher Chow groups, we have a commutative diagram with exact rows. The vertical maps are induced by $s$ via contravariant functoriality; we give them different names to distinguish among them in the sequel.

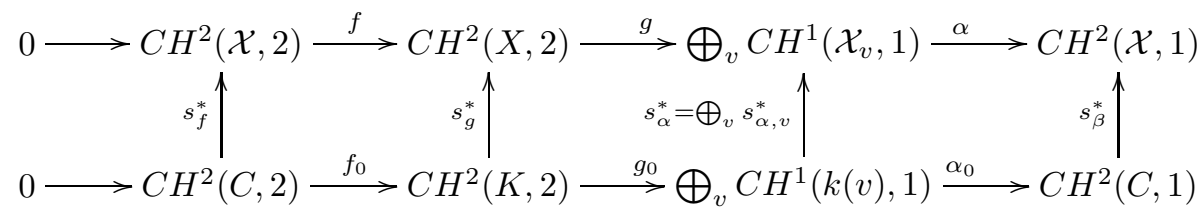

By the snake lemma, there is a long exact sequence

$$
0 \rightarrow \operatorname{Ker}\left(s^{*}: \operatorname{Im} g_{0} \rightarrow \operatorname{Im} g\right) \rightarrow \operatorname{Ker} s_{\alpha}^{*} \rightarrow \operatorname{Ker}\left(s^{*}: \operatorname{Im} \alpha_{0} \rightarrow \operatorname{Im} \alpha\right)
$$

$\rightarrow$ Coker $\left(s^{*}: \operatorname{Im} g_{0} \rightarrow \operatorname{Im} g\right) \rightarrow$ Coker $s_{\alpha}^{*} \rightarrow$ Coker $\left(s^{*}: \operatorname{Im} \alpha_{0} \rightarrow \operatorname{Im} \alpha\right) \rightarrow 0$.

Now, for each $v \in C$, we have $k(v)^{*} \hookrightarrow C H^{1}\left(\mathcal{X}_{v}, 1\right)$, even if $\mathcal{X}_{v}$ is not smooth. Since it is clear that this inclusion coincides with the map

$$
s_{\alpha, v}^{*}: k(v)^{*} \longrightarrow C H^{1}\left(\mathcal{X}_{v}, 1\right),
$$

it follows that $\operatorname{Ker} s_{\alpha}^{*}=0$. Thus, $\operatorname{Ker}\left(s^{*}: \operatorname{Im} g_{0} \longrightarrow \operatorname{Im} g\right)=0$.

Similarly, there is another long exact sequence

$$
\begin{aligned}
& 0 \longrightarrow \operatorname{Ker} s_{f}^{*} \longrightarrow \operatorname{Ker} s_{g}^{*} \longrightarrow \operatorname{Ker}\left(s^{*}: \operatorname{Im} g_{0} \longrightarrow \operatorname{Im} g\right) \\
& \longrightarrow \text { Coker } s_{f}^{*} \longrightarrow \text { Coker } s_{g}^{*} \longrightarrow \text { Coker }\left(s^{*}: \operatorname{Im} g_{0} \longrightarrow \operatorname{Im} g\right) \longrightarrow 0
\end{aligned}
$$

which collapses to

$$
0 \longrightarrow \text { Coker } s_{f}^{*} \longrightarrow W(X) \longrightarrow \text { Coker }\left(s^{*}: \operatorname{Im} g_{0} \longrightarrow \operatorname{Im} g\right) \longrightarrow 0 .
$$

We claim that the last term of this short exact sequence is finitely generated. By the first long exact sequence, it suffices to show that the terms

$$
\operatorname{Ker}\left(s^{*}: \operatorname{Im} \alpha_{0} \longrightarrow \operatorname{Im} \alpha\right) \text { and Coker } s_{\alpha}^{*}
$$

are finitely generated. In the original ladder diagram, the map $\alpha_{0}$ is surjective; this is because the next term in the localization sequence would be $C H^{2}(K, 1)=0$. Thus $\operatorname{Im} \alpha_{0}=C H^{2}(C, 1) \cong k_{0}^{*}$ ([Ak2], Theorem 3.1), which is a finite group. Furthermore, for $v \in \mathcal{R}, s_{\alpha, v}$ is an isomorphism; thus, Coker $s_{\alpha}^{*}$ is finitely generated by Lemma 3.5 .

Finally, we examine the six-term exact sequence induced by multiplication by $n$ on the last short exact sequence above:

$$
\begin{aligned}
& 0 \longrightarrow{ }_{n} \text { Coker }_{s}^{*} \longrightarrow{ }_{n} W(X) \longrightarrow{ }_{n}\left(\text { Coker }^{*}: \operatorname{Im} g_{0} \longrightarrow \operatorname{Im} g\right) \\
& \longrightarrow \text { Coker } s_{f}^{*} / n \longrightarrow W(X) / n \longrightarrow\left(\text { Coker } s^{*}: \operatorname{Im} g_{0} \longrightarrow \operatorname{Im} g\right) / n \longrightarrow 0
\end{aligned}
$$


Noting that Coker $s_{f}^{*} / n$ is finite by Lemma 4.1 the desired assertion follows.

We remark that finiteness of ${ }_{n}$ Coker $s_{f}^{*}$ also follows from Lemma 4.1 thus yielding a proof of the finiteness of ${ }_{n} W(X)$. However, a much more general result implies that the entire torsion subgroup of $W(X)$ is finite:

Theorem 4.4 (Raskind and Colliot-Thélène, [CTR], Prop. 1.15). Let L be a field finitely generated over its prime field, and let $Y$ be a projective variety, smooth over $L$. Then the torsion subgroup of $H_{Z a r}^{0}(Y, 2) / K_{2} L$ is finite.

We are now in a position to prove the main result:

Theorem 4.5. $W(X)$ is finitely generated if and only if $K_{2}(\mathcal{X})$ is finite.

Proof. Following the proof of Theorem 3.1 first apply the Riemann-Roch Theorem to deduce

$$
K_{2}(\mathcal{X}) \otimes \mathbb{Q} \cong \bigoplus_{i} C H^{i}(\mathcal{X}, 2)
$$

For reasons of dimension, $C H^{i}(\mathcal{X}, 2)=0$ for $i>4$ or $i<0$. Moreover, $C H^{0}(\mathcal{X}, 2)=0, C H^{1}(\mathcal{X}, 2)=0, C H^{3}(\mathcal{X}, 2)$ is a torsion group by Ak2, Prop. 4.2 and $C H^{4}(\mathcal{X}, 2)=0$ by [Ak1], Cor. 7.1. Hence it suffices to prove that $W(X)$ is finitely generated if and only if $\mathrm{CH}^{2}(\mathcal{X}, 2)$ is torsion.

From the proof of Proposition 4.3, there is an exact sequence

$$
0 \longrightarrow \text { Coker } s_{f}^{*} \longrightarrow W(X) \longrightarrow F \longrightarrow 0
$$

in which $F$ is a finitely generated group. Thus $W(X)$ is finitely generated if and only if the same is true of Coker $s_{f}^{*}$. Now $C H^{2}(C, 2)$ is finite by Theorem 4.2, so Coker $s_{f}^{*}=C H^{2}(\mathcal{X}, 2) / s_{f}^{*}\left(C H^{2}(C, 2)\right)$ is finitely generated if and only if $C H^{2}(\mathcal{X}, 2)$ is finitely generated. Finally, Lemma 4.1] shows that finite generation of $C H^{2}(\mathcal{X}, 2)$ is equivalent to its finiteness.

Corollary 4.6. Suppose $X=\Gamma \times_{k_{0}} k$, where $\Gamma$ is a smooth projective curve over $k_{0}$. Then $W(X)$ is finitely generated.

\section{EXTENDing The CONSTANT FIELD}

The description of the finiteness of $W(X) / n$ as a "weak Mordell-Weil theorem" prompts one to consider whether or not $W(X)$ is finitely generated when $X$ is a curve over some field $k$ for which the ordinary Mordell-Weil theorem holds - that is, for which the group $A(k)$ of $k$-rational points on an abelian variety is finitely generated. In this section, we show that if $\bar{k}_{0}$ is the algebraic closure of a finite field and $k_{c}$ is a finitely generated field of transcendence degree 1 over $\overline{k_{0}}$ (so that the ordinary Mordell-Weil theorem holds for $k_{c}$ ), there are examples of curves $X$ such that $W(X)$ is not finitely generated. We also study the group $V(X)$ for these fields.

Let $i: L_{1} \hookrightarrow L_{2}$ be a finite extension of fields. There are functorial maps $V(X) \stackrel{i^{*}}{\rightarrow} V\left(X_{L}\right)$ and $V\left(X_{L}\right) \stackrel{i_{*}}{\rightarrow} V(X)$ associated to the (flat and proper) morphism $X_{L} \longrightarrow X$ induced by base change; the projection formula for higher Chow groups then shows that $i_{*} \circ i^{*}$ is simply multiplication by the field degree $[L: k]$. Thus, to show that $V(X)$ is torsion, it is sufficient to show that $V\left(X_{L}\right)$ is torsion for any finite extension $L / k$. A standard argument using direct limits shows that it suffices to prove that $V\left(X_{\bar{k}}\right)$ is torsion, where $\bar{k}$ is an algebraic closure of $k$. Furthermore, 
Raskind ([R3], Lemma 1.1) has proved that $V\left(X_{\bar{k}}\right)$ is uniquely divisible, so in fact it suffices to show that $V\left(X_{\bar{k}}\right)=0$.

The disadvantage of working with $X_{\bar{k}}$ is that one cannot apply Abhyankar's Theorem. As a consolation, one might extend the constant field $k_{0}$ to an algebraic closure $\overline{k_{0}}$ and work with $k_{c}=k \overline{k_{0}}$; the generic fiber of the map $s_{\overline{k_{0}}}: \mathcal{X}_{\overline{k_{0}}} \longrightarrow C_{\overline{k_{0}}}$ obtained by base extension is then $X_{c}=X \times_{k} k_{c}$. Just as in the case of a finite constant field, one may apply the localization sequence to study $V\left(X_{c}\right)$; however, the finiteness results of Lemma 3.6 and Theorem 3.8 no longer hold. For instance, even when $\mathcal{X}_{\overline{k_{0}}}$ is a product $\Gamma \times \Gamma^{\prime}$ of curves over $\overline{k_{0}}, H_{Z a r}^{1}\left(\mathcal{X}_{\overline{k_{0}}}^{-}, \mathcal{K}_{2}\right) \cong C H^{2}\left(\mathcal{X}_{\overline{k_{0}}}, 1\right)$ contains $C H^{1}(\Gamma, 1) \cong{\overline{k_{0}}}^{*}$ which is an infinite (torsion) group.

Nonetheless, it is possible to make a few observations concerning $V\left(X_{c}\right)$. Since $k_{c}$ is finitely generated of transcendence degree 1 over the algebraically closed field $\overline{k_{0}}$, we see that every algebraic extension $L / k_{c}$ has cohomological dimension $\leq 1$, and hence for any finite extensions $M \supseteq L \supseteq k_{c}$, the field norm $N_{M / L}: M^{*} \longrightarrow L^{*}$ is surjective. We apply this principle in the following:

Proposition 5.1. The group $V\left(X_{c}\right)$ is divisible.

Proof. Fix a nonzero integer $n$. The group $V\left(X_{c}\right) \subseteq C H^{2}\left(X_{c}, 1\right)$ is generated by elements of the form $N_{L / k}(z \cdot a)$, where $z \in A_{0}\left(X_{L}\right)=\operatorname{Ker}\left(\operatorname{deg}: C H_{0}\left(X_{L}\right) \rightarrow \mathbb{Z}\right) \subseteq$ $C H^{1}\left(X_{L}\right), a \in L^{*} \cong C H^{1}(L, 1)$ for some finite extension $L / k, \cdot$ is the intersection product on higher Chow groups, and $N_{L / k}$ is the covariant map on higher Chow groups. Noting that $A_{0}\left(X_{\bar{k}}\right)$ is divisible by a result of Bloch (B11], Lemma 1.3), choose an extension $i: L \hookrightarrow M$ such that $i^{*} z=n y$ for some $y \in A_{0}\left(X_{M}\right)$ and select $b \in M^{*}$ such that $N_{M / L} b=a$. Then by the projection formula,

$$
N_{L / k}(z \cdot a)=N_{L / k}\left(z \cdot N_{M / L} b\right)=N_{L / k} N_{M / L}\left(i^{*} z \cdot b\right)=n N_{M / k}(y \cdot b) .
$$

We remark that this result follows directly from the interpretation of $V\left(X_{c}\right)$ as the generalized Milnor $K$-group $K\left(k ; J ; \mathbf{G}_{m}\right)$ in which $J$ is the Jacobian variety of the curve $X_{c}$ and $\mathbf{G}_{m}$ is the mutiplicative group scheme (cf. [Som], Theorem 2.1 and [Ak3], Theorem 3.1).

We now turn our attention to the group $W\left(X_{c}\right)$. The rest of the discussion is devoted to the exposition of an example illustrating that this group is not always finitely generated.

Proposition 5.2. Let $C$ be a smooth projective curve over $k_{0}$ of genus $g$ and $X=C_{k(C)}$. If $g>0$, then $W\left(X_{c}\right)$ is not finitely generated.

Note that in this case $\mathcal{X}=C \times_{k_{0}} C$. For convenience of notation, set $\bar{C}=$ $C \times_{k_{0}} \overline{k_{0}}$. Thus $\mathcal{X} \times_{k_{0}} \overline{k_{0}} \cong \bar{C} \times_{\overline{k_{0}}} \bar{C}$.

For the proof, we will need the following result of Colliot-Thélène and Raskind, which was, historically, the precursor of Lemma 4.1.

Lemma 5.3 (Colliot-Thélène, Raskind [CTR]). Let $F$ be a separably closed field and $Y$ a smooth proper connected variety over $F$. Let $p$ be the characteristic of $F$. Then there is an exact sequence

$$
0 \longrightarrow D \longrightarrow H_{Z a r}^{0}\left(Y, \mathcal{K}_{2}\right) \longrightarrow \bigoplus_{l \neq p}{ }_{l} H_{e t}^{2}\left(Y, \mathbf{Z}_{l}(2)\right) \longrightarrow 0
$$

in which $D$ is divisible prime to the characteristic of $F$ and the group on the right is finite. 
Furthermore, for $l \neq p$ and any $m \geq 0$, there is a natural isomorphism

$$
l^{m} \operatorname{Pic}^{0}(Y) \otimes \mu_{l^{m}} \cong l^{m} D .
$$

Proof of Proposition 5.2, Let $\pi: \bar{C} \times{ }_{k_{0}} \bar{C}$ be the first projection map. Then the map $\pi^{*}: C H^{2}(\bar{C}, 2) \longrightarrow C H^{2}\left(\bar{C} \times_{\overline{k_{0}}} \bar{C}, 2\right)$ is a split injection; hence there is an exact sequence

$$
0 \rightarrow{ }_{n} C H^{2}(\bar{C}, 2) \stackrel{\pi^{*}}{\rightarrow}{ }_{n} C H^{2}\left(\bar{C} \times{ }_{\overline{k_{0}}} \bar{C}, 2\right) \rightarrow{ }_{n} \text { Coker } \pi^{*} \rightarrow 0 .
$$

We claim that ${ }_{n}$ Coker $\pi^{*}$ is nonzero for infinitely many $n$. Now if $n$ is a prime power relatively prime to $p$, to the order of $\bigoplus_{l \neq p}{ }_{l} H_{e t}^{2}\left(\bar{C}, \mathbf{Z}_{l}(2)\right)$, and to the order of $\bigoplus_{l \neq p}{ }_{l} H_{e t}^{2}\left(\bar{C} \times \overline{k_{0}} \bar{C}, \mathbf{Z}_{l}(2)\right)$, then by Lemma $[5.3]$ we have ${ }_{n} C H^{2}(\bar{C}, 2) \cong(\mathbb{Z} / n \mathbb{Z})^{2 g} \otimes$ $\mu_{n}$ whereas ${ }_{n} C H^{2}\left(\bar{C} \times \overline{k_{0}} \bar{C}, 2\right) \cong(\mathbb{Z} / n \mathbb{Z})^{4 g} \otimes \mu_{n}$. Therefore ${ }_{n}$ Coker $\pi^{*} \neq 0$ for all such $n$.

Finally, by reasoning identical to that used in the proof of Proposition 4.3, there is an exact sequence

$$
0 \longrightarrow \text { Coker } \pi^{*} \longrightarrow W\left(X_{c}\right) \longrightarrow A \longrightarrow 0
$$

for some group $A$. This shows that ${ }_{n}$ Coker $\pi^{*}$ injects into ${ }_{n} W\left(X_{c}\right)$, hence our conclusion.

\section{REFERENCES}

[Ab] Abhyankar, S. Resolution of singularities for arithmetical surfaces, in Arithmetical algebraic geometry, O. Schilling (ed.) Harper and Row 1963. MR0200272 (34:171)

[Ak1] Akhtar, R. Milnor $K$-theory of smooth varieties. $K$-theory 32 (2004), 269-291.

[Ak2] Akhtar, R. Zero-cycles on varieties over finite fields. Comm. Alg. 32 (2004), no. 1, 279294. MR2036237|(2005b:14017)

[Ak3] Akhtar, R. Torsion in mixed $K$-groups. Comm. Alg. 32 (2004), no. 1, 295-313. MR 2036238

[BT] Bass, H. and Tate, J. The Milnor ring of a global field. Springer Lecture Notes in Math. 342 (1973), 349-446. MR0442061 (56:449)

[Bl1] Bloch, S. Lectures on Algebraic Cycles. Duke University Mathematics Series, IV. Duke University, 1980. MR0558224 (82e:14012)

[B12] Bloch, S. Algebraic $K$-theory and classfield theory for arithmetic surfaces. Ann. of Math. (2) 114 (1981), no. 2, 229-265. MR0632840 (83m:14025)

[Bl3] Bloch, S. Algebraic cycles and higher $K$-theory, Adv. Math. 61 (1986), 267-304. MR.0852815 (88f:18010)

[BL] Bloch, S. and Lichtenbaum, S. A spectral sequence for motivic cohomology. Preprint.

[CTR] Colliot-Thélène, J-L. and Raskind, W. $\mathcal{K}_{2}$-cohomology and the second Chow group. Math. Ann. 270 (1985), no. 2, 165-199. MR0771978 (86m:14005)

[CTSS] Colliot-Thélène, J-L., Sansuc, J-J. and Soulé, Christophe. Torsion dans la groupe de Chow de codimension deux. Duke Math. J. 50 (1983), no. 3, 763-801. MR0714830 (85d:14010)

[FS] Friedlander, E. and Suslin, A. The spectral sequence relating algebraic $K$-theory to motivic cohomology. Ann. Sci. Ecole Norm. Sup. (4) 35 (2002), no. 6, 773-875. MR1949356 (2004b:19006)

[Ge] Geisser, T. Tate's Conjecture, Algebraic Cycles and Rational $K$-Theory in Characteristic p. $K$-theory 13, no.2, (1998), 109-122. MR1611623 (99c:19003)

[GS] Gros, M. and Suwa, N. Application d'Abel-Jacobi $p$-adique et cycles algébriques. Duke Mathematical Journal 55 (1988), no.2, 579-613. MR0962521|(89h:14006a)

[KS] Kato, K. and Saito, S. Unramified class field theory of arithmetic surfaces. Annals of Math. 118 (1983), 241-275. MR0717824 (86c:14006)

[MSEV] Müller-Stach, Stefan and Elbaz-Vincent, Phillippe. Milnor $K$-theory of rings, higher Chow groups and applications. Invent. Math. 148 (2002), no. 1, 177-206. MR1892848 (2003c:19001) 
[Ne] Nestler, A. Ph.D. thesis. University of Southern California, 2000.

[Qu1] Quillen, D. On the cohomology and $K$-theory of the general linear group over a finite field. Ann. of Math. (2) 96 (1972), 552-586. MR0315016 (47:3565)

[Qu2] Quillen, D. Higher Algebraic K-theory I. Springer Lecture Notes in Math. 341 (1973), 85-147. MF 0338129 (49:2895)

[R1] Raskind, W. "Le théorème de Mordell-Weil faible" pour $H^{0}\left(X, \mathcal{K}_{2}\right) / K_{2} k$. C. R. Acad. Sci. Paris I Math. 299 (1984), no. 7, 241-244. MR0762730 (86a:14017)

[R2] Raskind, W. Algebraic K-theory, etale cohomology and torsion algebraic cycles, in Algebraic $K$-theory and algebraic number theory (Honolulu, HI, 1987), Contemp. Math. 83 (1989), 311-341. MR0991983 (90d:14011)

[R3] Raskind, W. On $K_{1}$ of curves over global fields. Math. Ann. 288 (1990), no. 2, 179-193. MR,1075763 (91m:14013)

[Sha] Shapiro, J.M. Relations between the Milnor and Quillen $K$-theory of fields. J. Pure and Applied Algebra. 20 (1981), 93-102. MR0596156 (82f:12016)

[She] Sherman, C. Some theorems on the $K$-theory of coherent sheaves. Comm. Algebra 7 (1979), no. 14, 1489-1508. MR0541048 (80k:14029)

[Som] Somekawa, M. On Milnor $K$-groups attached to semiabelian varieties. $K$-theory 4 (1990), 105-119. MR1081654 (91k:11052)

[Sou] Soulé, C. Groupes de Chow et $K$-théorie. Math. Annalen 268, vol 1 (1984), 317-345. MR.0751733 (86k:14017)

Department of Mathematics and Statistics, Miami University, Oxford, Ohio 45056

E-mail address: reza@calico.mth.muohio.edu 